\title{
Interpolation of externally-caused magnetic fields over large sparse arrays using Spherical Elementary Current Systems
}

\author{
S. A. McLay ${ }^{1}$ and C. D. Beggan ${ }^{2}$ \\ ${ }^{1}$ School of GeoSciences, University of Edinburgh, West Mains Road, Edinburgh, EH9 3JW, UK \\ ${ }^{2}$ British Geological Survey, Murchison House, West Mains Road, Edinburgh, EH9 3LA, UK
}

Received: 22 July 2010 - Revised: 25 August 2010 - Accepted: 15 September 2010 - Published: 30 September 2010

\begin{abstract}
A physically-based technique for interpolating external magnetic field disturbances across large spatial areas can be achieved with the Spherical Elementary Current System (SECS) method using data from ground-based magnetic observatories. The SECS method represents complex electrical current systems as a simple set of equivalent currents placed at a specific height in the ionosphere. The magnetic field recorded at observatories can be used to invert for the electrical currents, which can subsequently be employed to interpolate or extrapolate the magnetic field across a large area. We show that, in addition to the ionospheric currents, inverting for induced subsurface current systems can result in strong improvements to the estimate of the interpolated magnetic field. We investigate the application of the SECS method at mid- to high geomagnetic latitudes using a series of observatory networks to test the performance of the external field interpolation over large distances. We demonstrate that relatively few observatories are required to produce an estimate that is better than either assuming no external field change or interpolation using latitudinal weighting of data from two other observatories.
\end{abstract}

Keywords. Geomagnetism and paleomagnetism (Rapid time variations) - Ionosphere (Electric fields and currents; Modeling and forecasting)

\section{Introduction}

The geomagnetic field measured on the surface of the Earth is composed of temporally and spatially varying components. The primary sources include (1) the main field generated in the outer core, (2) the crustal field and (3) fields produced by solar-terrestrial interaction. Electrical currents

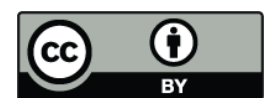

\section{Correspondence to: C. D. Beggan} (ciar@bgs.ac.uk) induced in the subsurface are a secondary source contribution to the measured magnetic field, but can be significant. Any surface measurement is a summation of these different fields at each instant of time. On time periods of seconds to days, changes in the magnetic field are principally of external origin and are caused by the solar wind, magnetosphere and ionosphere/upper atmosphere interaction (e.g. Campbell, 2003).

The study of temporal changes in the geomagnetic field and the accompanying induced electric fields are of interest in Earth hazard research. There are two classes of technology that are adversely impacted by space weather: those directly affected by variations in the geomagnetic field and those affected by the electric currents induced by the changing field. Modern applications of space weather studies to ground-based technology consider the problems of geomagnetically induced currents within power grids, oil and gas pipelines, telecommunication cables and railway equipment (Boteler et al., 1998; Pirjola et al., 2000; Pirjola, 2005). Indirectly, geomagnetic disturbances in the ionosphere/upper atmosphere can disrupt the operation of technology exploiting real-time satellite data, such as positioning and navigation systems (e.g. Klobuchar, 1986). Estimates of external magnetic field values are often required at locations where it may not be possible or practical to make accurate measurements. For example, hydrocarbon exploration and production often demands real-time geomagnetic reference data at offshore oilfields around the UK and at other high latitude regions (Reay et al., 2005). Using real-time external field data aids accuracy in positional control of directional boreholes for safer extraction of oil and gas in challenging reservoirs (Bowe and McCulloch, 2007).

The surface effects of the external magnetic field can be modelled in terms of ionospheric equivalent currents. Complex 3-D currents systems may be represented as simple spherical sheets of current which mimic the magnetic effects of the ionosphere (Vanhamäki et al., 2003). Telluric currents

Published by Copernicus Publications on behalf of the European Geosciences Union. 
induced in the subsurface can also produce significant magnetic field disturbances. During sub-storm onset their contribution can be up to $40 \%$ of the total observed change (Tanskanen et al., 2001). The magnetic effects of telluric currents can also be modelled as equivalent currents. These can be constructed to lie within the subsurface and the superposition of their magnetic effect with the ionospheric contribution can be used to calculate the total ground magnetic field disturbance.

Several techniques have been developed to calculate equivalent currents from ground-based measurements; two of the most successful thus far have been the Fourier method (Mersmann et al., 1979) and Spherical Cap Harmonic Analysis (SCHA) (Haines, 1985). The Fourier method assumes planarity of the Earth's surface which limits its spatial applicability, while the SCHA method employs global wavelength cut-offs and consequently is prone to aliasing effects, particularly with noisy data. A third approach, the Spherical Elementary Current System (SECS) method, was established and developed by Amm (1997) and Amm and Viljanen (1999) respectively. The use of SECS overcomes some of the limitations of the other two methods and is suitable for studies both on a local and global scale. Amm and Viljanen (1999) demonstrated that the SECS technique produced a better fit than SCHA to simulated ionosphere conditions, while Pulkkinen et al. (2003b) showed it to be applicable for the interpolation of ionospheric fields in a densely sampled, relatively small region of northern Scandinavia.

In this paper we use the SECS method to interpolate external magnetic field disturbances over large spatial areas using relatively few input observatories. We test the method with data from networks of magnetometers in North America, around the North Sea and in Central Europe. The ability to model large and complex current variations in the ionosphere is investigated, as well as the adaptability to different spatial network configurations and scales. In Sect. 2 we briefly cover the theory of SECS and the computational framework for the interpolation of magnetic field disturbances. In Sect. 3 we describe the data used in the study and in Sect. 4 we illustrate the resulting interpolation from the SECS method by comparison to measured observatory data. We discuss the applications and limitations of the technique in Sect. 5.

\section{Spherical Elementary Current Systems}

Ionospheric currents form a complex 3-D system, comprising three distinct current flows: Hall, Pedersen and fieldaligned currents (FACs) (Ritter et al., 2004). By assuming sets of uniform conductance within the ionosphere and by using Ampere's law, Fukushima (1976) first showed that magnetic perturbations produced by Pederson currents and FACs cancel each other below the base of the ionosphere. As the curl-free part of the current system $\mathbf{J}_{\mathrm{cf}}$ is associated with FACs it will produce no magnetic effect below the de- fined base of the ionosphere. (Note, this property is actually valid independently of how the currents are produced, i.e. it does not depend on the type of current flowing in the ionosphere or whether the conductances are uniform or not.) The curl-free system and therefore measurements at the surface of the Earth can neglect these effects. As a result of this simplification, ground magnetic field disturbances can be obtained from an exclusively divergence-free system of equivalent currents at a specific height in the ionosphere. A similar approach can be taken in the subsurface, by placing the elementary current systems at an appropriate depth. It is important to remember that ionospheric equivalent currents are not a true representation of the 3-D current systems in the ionosphere or in the subsurface, but are 2-D current sheets that produce a similar magnetic effect.

\subsection{Background}

The basic concept of SECS is to construct the equivalent current system using a linear superposition of divergence-free elementary current systems, all of which can be placed freely within the current plane. Amm (1997) defined a divergencefree system of spherical elementary sheet currents, $\mathbf{J}_{\mathrm{df}}$, using a spherical coordinate system $(r, \theta, \phi)$ with unit vectors $\left(\boldsymbol{e}_{r}, \boldsymbol{e}_{\theta}, \boldsymbol{e}_{\phi}\right)$ such that the poles of the elementary systems are at their centres. With this description current sheets can be defined for the ionosphere and the subsurface:

$\mathbf{J}_{\mathrm{df}}^{\mathrm{ext}}(r, \theta)=\frac{I^{\mathrm{e}}}{4 \pi R_{\mathrm{S}}} \cot \left(\frac{\theta}{2}\right) \boldsymbol{e}_{\phi}$
$\mathbf{J}_{\mathrm{df}}^{\mathrm{int}}(r, \theta)=\frac{I^{\mathrm{i}}}{4 \pi R_{\mathrm{G}}} \cot \left(\frac{\theta}{2}\right) \boldsymbol{e}_{\phi}$

In this description $I^{\mathrm{e}}$ and $I^{\mathrm{i}}$ are the scaling factors for both external and internal divergence-free current systems and $R_{\mathrm{S}}$ and $R_{\mathrm{G}}$ are the radius of the ionosphere and the depth of inductance in the ground, respectively. These radii are defined in this study as infinitely thin layers $110 \mathrm{~km}$ above the Earth's surface and $100 \mathrm{~km}$ beneath the Earth's surface. By applying Helmholtz's theorem to Eqs. (1) and (2), Amm (1997) demonstrated that any ionospheric current distribution may be constructed uniquely by placing the poles of elementary systems within a plane.

The method developed in Amm and Viljanen (1999) introduced the continuation of the magnetic field disturbance from the ground to the ionosphere using SECS placement. Pulkkinen et al. (2003a) extended the complex image method of Pirjola and Viljanen (1998) to show that the field could be calculated from superposition of the magnetic effect of two horizontal current layers composed of divergence-free elementary current systems. Their derivation is constructed for a point at radius $r$ between the current system in the ground and in the ionosphere, $R_{\mathrm{G}}<r<R_{\mathrm{S}}$ for a position $(\theta, \phi)$ on the Earth. The magnetic field vector $(\boldsymbol{B})$ at the surface of the Earth is then expressed by the superposition of the magnetic 
effect of these two layers of horizontal currents consisting of a series of elementary current systems:

$$
\begin{aligned}
\boldsymbol{B}(r, \theta, \phi) & =\sum_{j=1}^{L} I_{j}^{\mathrm{i}} T_{\mathrm{df}}^{\mathrm{i}}\left(R_{\mathrm{G}}, \theta_{j}, \phi_{j}, r, \theta, \phi\right) \\
& +\sum_{k=1}^{M} I_{k}^{\mathrm{e}} T_{\mathrm{df}}^{\mathrm{e}}\left(R_{\mathrm{S}}, \theta_{k}, \phi_{k}, r, \theta, \phi\right)
\end{aligned}
$$

where $T_{\mathrm{df}}^{\mathrm{e}}$ and $T_{\mathrm{df}}^{\mathrm{i}}$ are the geometric parts of the external and internal magnetic fields produced by each elementary current system located at $\left(R_{\mathrm{G}}, \theta_{j}, \phi_{j}\right)$ and $\left(R_{\mathrm{S}}, \theta_{k}, \phi_{k}\right) . L$ and $M$, with subscripts $j$ and $k$, denote the number of current systems solved for within the ground and at the radius of the ionosphere, respectively. Formulation of the magnetic field superposition is given in Appendix A of Pulkkinen et al. (2003a).

\subsection{Inversion for current system scalings}

We assume that the magnetic field vector $\boldsymbol{B}$ has been measured at a set of points and construct a linear system of equations relating the measured field to the geometric parts and scaling factors of both the internal and external elementary current systems. Expressing this in matrix form gives:

$\mathbf{B}=\mathbf{T} \cdot \mathbf{I}$

We look to solve the linear inverse problem to determine the scaling factors $I$ of the internal and external elementary current systems. Due to the (generally) limited number of fixed ground magnetic observatories, the number of observation points is usually much lower than the number of elementary current systems required to produce a good representation of the actual currents. The linear inverse problem is therefore highly underdetermined:

$\mathbf{I}=\mathbf{T}^{-1} \cdot \mathbf{B}$

The matrix $\mathbf{T}$ may be badly conditioned which produces numerical instabilities when attempting to invert Eq. (5) directly. Thus, we employ Singular Value Decomposition (SVD) for the inversion, truncating such that any eigenvalues with an absolute value less than 1/100th of the largest are excluded from the solution, as suggested in Pulkkinen et al. (2003b). The truncation stabilises the inversion and tends to produce a slight smoothing effect on the solution. Other truncation levels were tested, including the truncation of eigenvalues with an absolute value less than 1/10th of the largest value and also no truncation. The results were stable and indeed quite similar; suggesting in these experiments that the T matrix is not actually badly conditioned.

We solve for the scaling factors of the currents systems on a rectangular grid evenly-spaced in latitude and longitude (note, any reasonable shape and spacing could be used). The forward solution for the magnetic field $(\boldsymbol{B})$ on the Earth's surface at any position within the grid can be calculated by determining the $\mathbf{T}$ matrix for the point of interest and using the scaling factors from the inversion.

Modelling was undertaken using minute mean magnetic field vector values. To prepare the data for inversion, the internal and crustal fields for each day were removed by subtracting the mean daily baseline values from the $\mathrm{X}, \mathrm{Y}$ and $\mathrm{Z}$ components, giving a 24-h set of magnetic disturbance values about the average. This deviation from the mean was used as the input into the $\mathbf{B}$ matrix and the positions of the observatories were used to construct the $\mathbf{T}$ matrix. The scaling factors (I) for the current systems across the grid of points were solved for every minute of the day.

The accuracy of the interpolation method is quantified by comparing the estimated magnetic field to data measured at an observatory which has not been used in the inversion. The root-mean-square (RMS) error (or misfit) between the estimated magnetic field and the measured field at time $t$ can be calculated as:

$\mathrm{RMS}=\sqrt{\frac{1}{N} \sum_{t}^{N}\left(\boldsymbol{B}_{\mathrm{obs}}^{t}-\boldsymbol{B}_{\mathrm{SECS}}^{t}\right)^{2}}$

where the subscript "obs" indicates the measured field at an observatory and "SECS" is the estimated field, for each of the three components of the magnetic field vector. The power of the measured data is described by the root-mean-square of the values, giving a coarse estimate of how magnetically disturbed the day was:

Power $=\sqrt{\frac{1}{N} \sum_{t}^{N}\left(\boldsymbol{B}_{\mathrm{Obs}}^{t}\right)^{2}}$.

\section{Investigation of controlling factors}

We wish to investigate how well the SECS method can interpolate the magnetic field across a region. It is assumed that the goodness of fit of the interpolation depends on several factors, including region size, the number of input observatories, and the relative spacing and location of the observatories. We also investigate the effect of relatively magnetically disturbed versus relatively quiet days, particularly in terms of the magnitude of the changes due to the external field and whether there are obvious differences e.g. due to the longitude offset of the observatories or perhaps induced currents.

We constructed five networks of magnetic observatories at mid- to high geomagnetic latitudes, with the number of input observatories varying from five to eight. Networks 1, 2 and 3 use eight, five and seven observatories, respectively, located at high geomagnetic latitude within the auroral zone in North America $\left(\approx 60^{\circ}-72^{\circ} \mathrm{N}\right)$. Network 4 uses data from four observatories and four directionally uncalibrated variometers placed around the North Sea. Network 5 located in the mid-latitude region on the European continent employs 

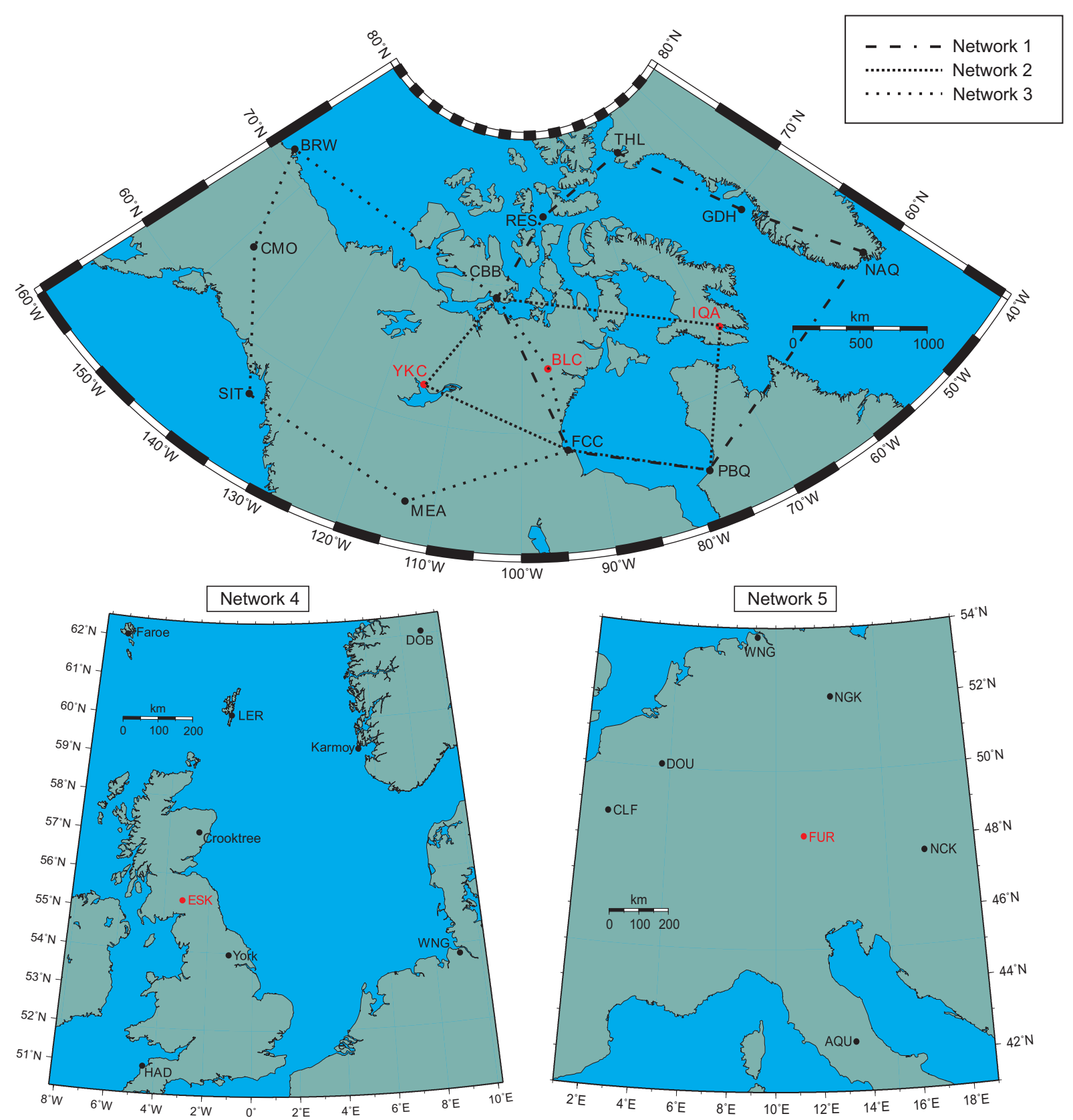

Fig. 1. Geographical arrangement of the five networks of magnetic observatories used in this study. The observatories used for comparison (denoted in red) for each network are: Network 1: IQA (Iqaluit); Network 2: BLC (Baker Lake); Network 3: YKC (Yellowknife); Network 4: ESK (Eskdalemuir); Network 5: FUR (Furstenfeldbruck). Observatories are denoted with the upper-case IAGA three-letter code. Variometers in Network 4 are denoted by their full name.

six observatories. Figure 1 shows the regions and observatories used in the study. Observatories denoted in red are excluded from the inversion and are subsequently used to examine how well the SECS method has estimated the field at their location. For each network, a rectangular grid of elementary current systems evenly-spaced in latitude and longitude was constructed. In Networks 1-3, the grid spacing was $2^{\circ}$, while a spacing of $0.5^{\circ}$ was used in Networks 4 and 5 as they cover smaller areas. Through preliminary experimentation, it was found that estimates of the magnetic field are relatively insensitive to the elementary system grid spacing. A finer grid enhances the nullspace of the solution (as the problem is generally underdetermined) and so the inversion using SVD will still separate the solution correctly. 

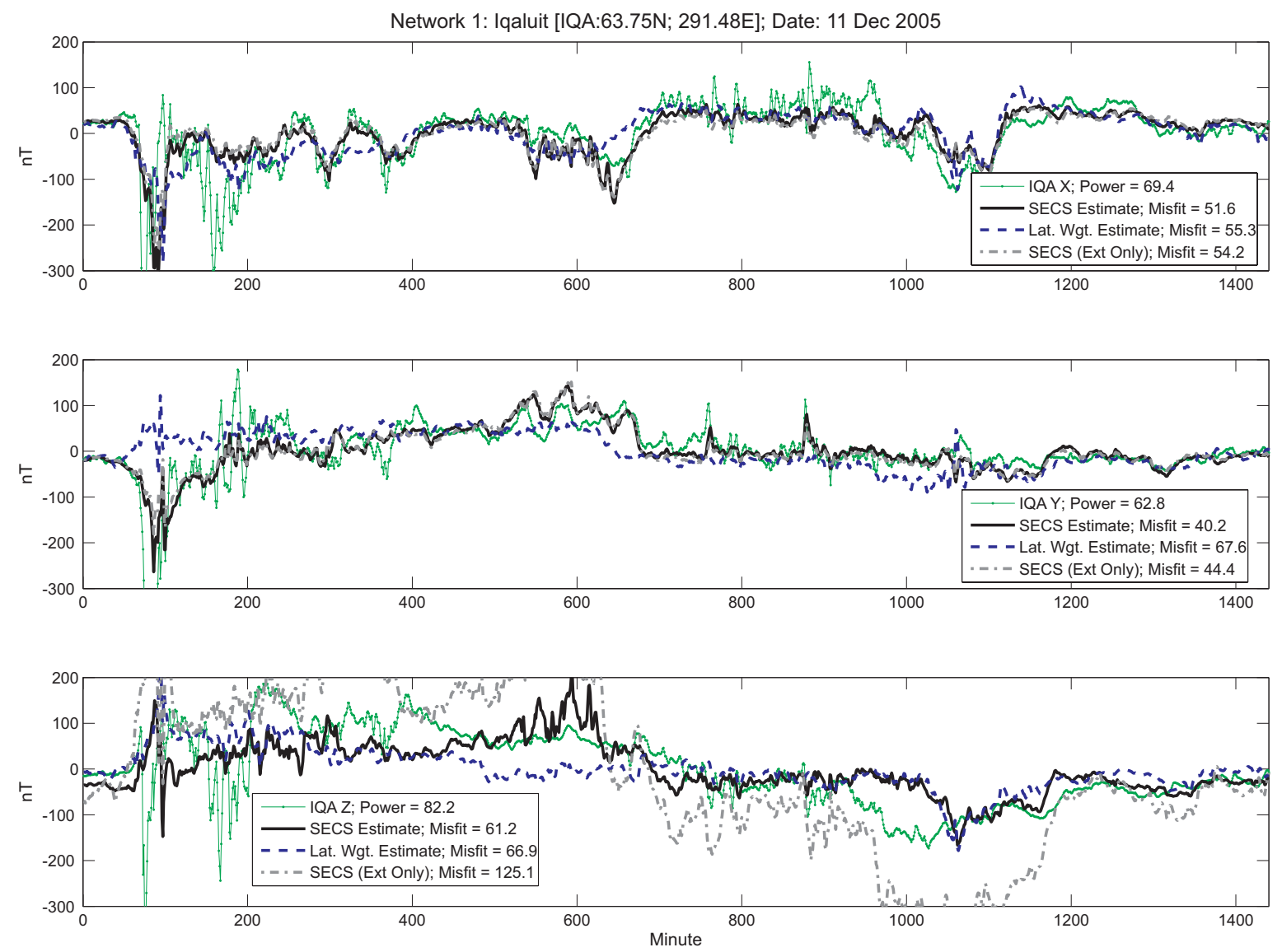

Fig. 2. Comparison of the measured external field disturbance (green solid, dots) and the estimate from SECS (black solid), Latitudinal Weighted (blue dashed) and SECS (External only) (grey dot-dash) at magnetic observatory Iqaluit (IQA) during a magnetically disturbed day (11 December 2005). Power and RMS misfit are in nT. Y-axis scaled to show finer details.

The magnetic field data were sourced from the World Data Centre for Geomagnetism (Edinburgh) and the International Real-time Magnetic Observatory Network (INTERMAGNET) (Kerridge, 2001). The variometer data used in Network 4 were acquired from the UK Sub-Auroral Magnetometer Network (SAMNET) data repository.

In addition, we tested two alternative implementations of the SECS equation. We solved solely for the external current elementary system scale value $\left(I^{\mathrm{e}}\right)$, thus excluding the internal current systems $\left(I^{\mathrm{i}}\right)$. The external-only SECS (second term of Eq. 3) requires a solution for only half the number of parameters, and produces a different set of scaling factors compared to the solution using both the external and internal elementary current systems. We also solved for the $\mathrm{X}$ and $\mathrm{Y}$ components of the external field to test if the solution improved when the $\mathrm{Z}$ component was ignored.

A further interpolation method was also used as a comparison to the interpolation of the SECS method. We employed the latitudinal-weighted average of measurements from two observatories, one to the south and one to the north of the test observatory. In some networks, the two other observatories were not sited at a similar longitude to the test observatory, leading to interpolation errors due to differences in local time, for example. Hence, the resulting error in the interpolation is strongly dependent on the relative positions of the two observatories, in both latitude and longitude.

\section{Results}

We examined how well the SECS method performed with two separate experiments. Firstly, a magnetically disturbed day and a magnetically quiet day were chosen for detailed study and the estimate from SECS, SECS (External only) and latitudinal weighting were compared to data from an observatory. Secondly, three months of data were used to examine how well the three methods compared on average to the assumption that the external field did not change (i.e. predicting zero disturbance throughout the day). 

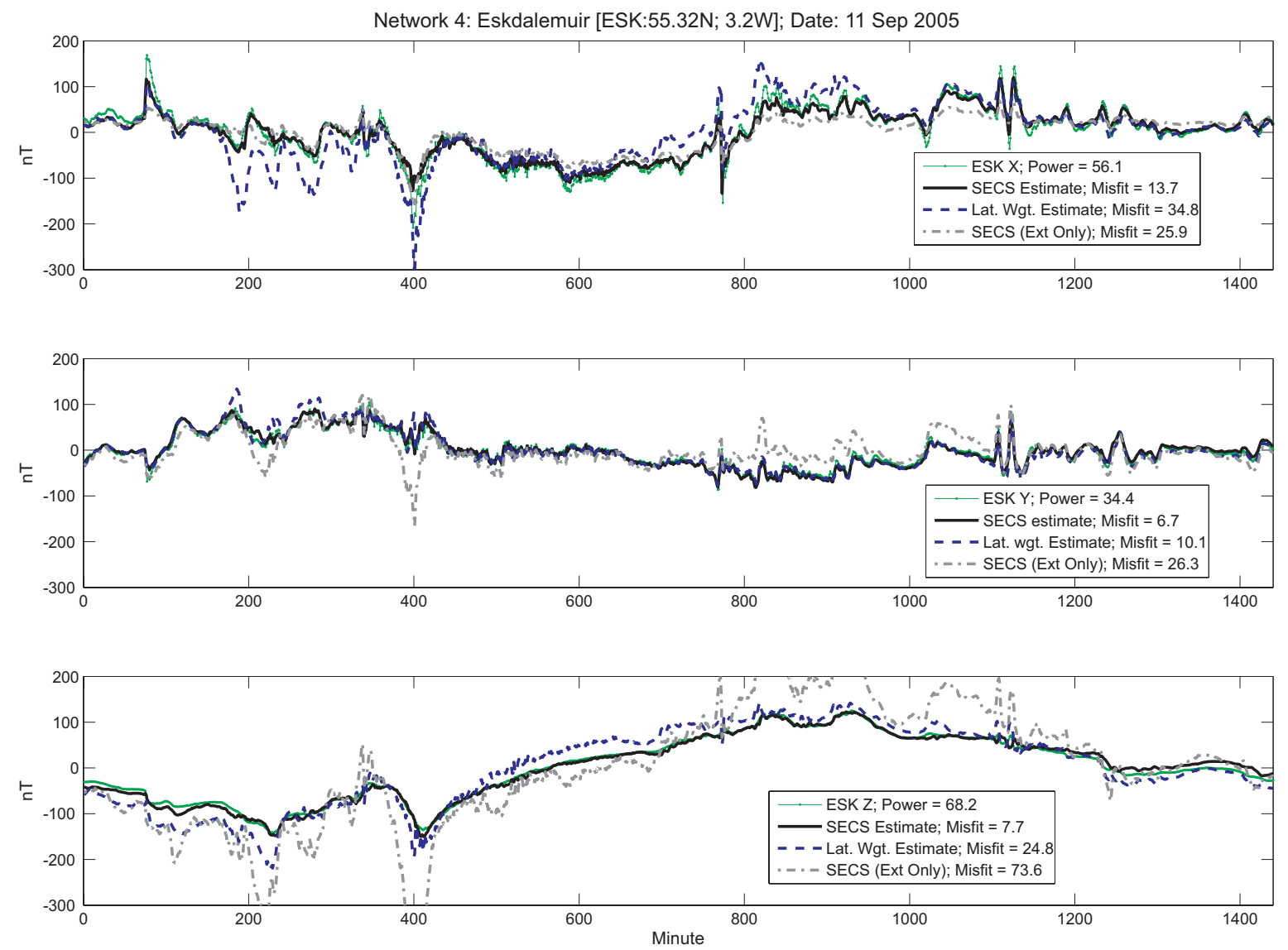

Fig. 3. Comparison of the measured external field disturbance (green solid, dots) and the estimate from SECS (black solid), Latitudinal Weighted (blue dashed) and SECS (External only) (grey dot-dash) at magnetic observatory Eskdalemuir (ESK) during a magnetically disturbed day (11 September 2005). Power and RMS misfit are in nT. Y-axis scaled to show finer details.

\subsection{Magnetically disturbed vs. quiet day}

For Networks 1-3, a magnetically disturbed day (11 December 2005) with $\mathrm{Kp}$ of up to 5- and a magnetically quiet day (7 December 2005) with a Kp of 0 were chosen. Due to the availability of data for Networks 4 and 5, different days were selected: a magnetically disturbed day (11 September 2005) with Kp of up to 7- and a magnetically quiet day (21 September 2005) with a Kp of 1+ were used instead. The results on the magnetically disturbed days for Network 1 and Network 4 are given in Figs. 2 and 3.

Figure 2 shows the comparison of the measured data at the Iqaluit (IQA) observatory (green solid, dots) and the estimate of the magnetic field from the SECS method using internal and external current systems (black solid) for Network 1. The estimate from interpolation using latitudinal weighting (blue dashed) of data from Qeqertarsuaq (GDH) and Narsarsuaq (NAQ) are also shown. Note both of these observatories are approximately $1000 \mathrm{~km}$ east of IQA. The SECS (External only) estimate solving only for an external set of current systems (grey dot-dash) is also plotted. The es- timate from inverting the SECS equation with only the $X$ and Y components was almost identical to the result for the SECS method using internal and external current systems and so is not plotted to avoid cluttering the figures.

Deviations of over $300 \mathrm{nT}$ in each component were recorded during the course of the day. The SECS estimation for the $\mathrm{X}, \mathrm{Y}$ and $\mathrm{Z}$ components of the magnetic field are relatively close to the measured data and are a better fit than the estimate from the latitudinal weighting in each of the components. However, the magnitude of large disturbances are sometimes underestimated (e.g. between minutes 50 and 250 , in all components) and the method does not manage to reproduce sharp spikes seen in the ground disturbance; rather the SECS estimate smoothes the variations in this part. An overestimation of the magnetic disturbance is also observed between minutes 425 and 650, particularly in the $\mathrm{Z}$ component. The SECS estimate manages to capture the gross features of the data throughout the course of the day. In the $X$ and Y components, the SECS (External only) estimate compares very well with the SECS estimate but is extremely poor in the $\mathrm{Z}$ component. 
Network 1: Iqaluit [IQA:63.75N; 291.482E]
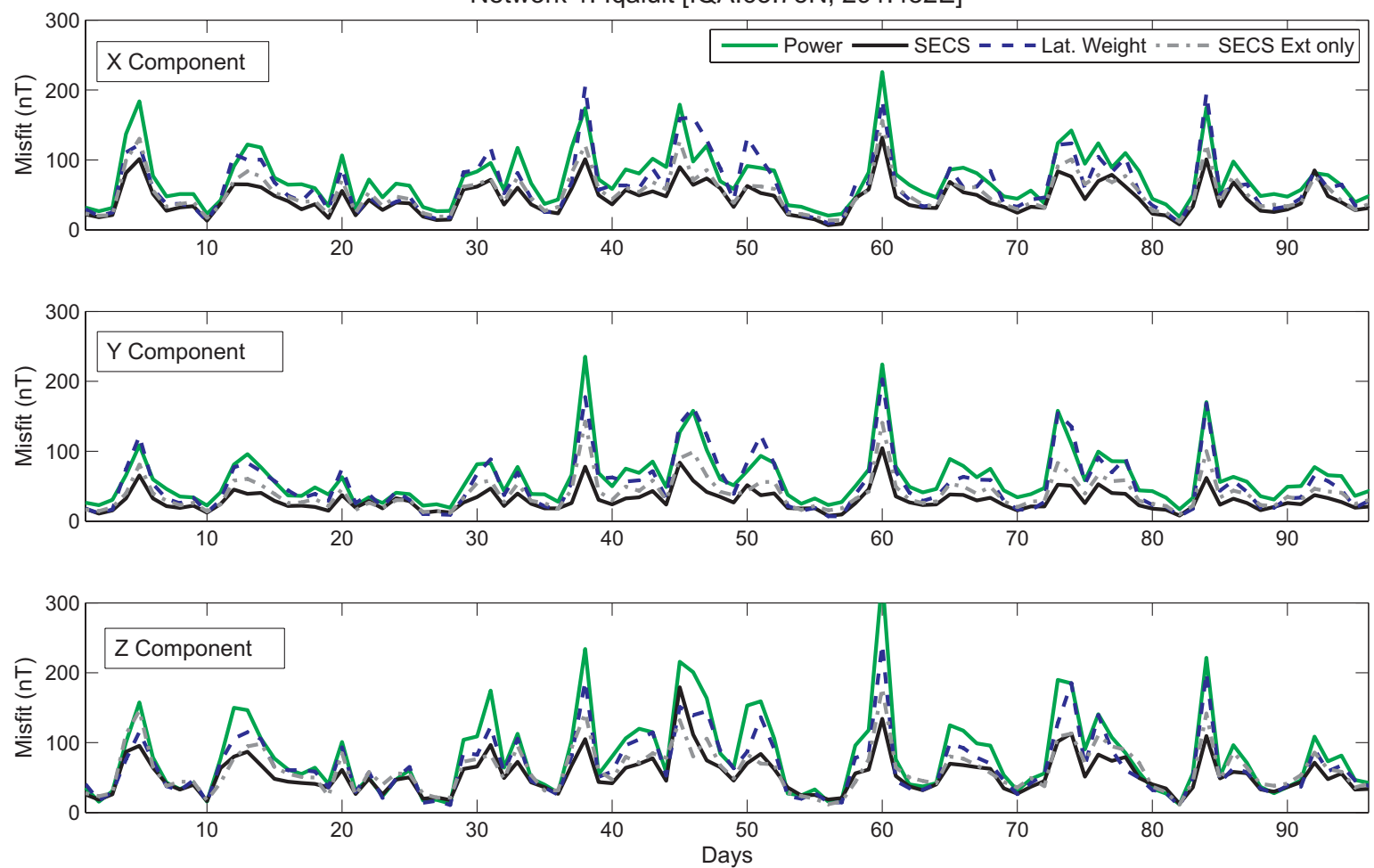

Fig. 4. Comparison of the daily power of measured external disturbances (green solid) with the misfit estimate from the SECS (black solid), Latitudinal Weighted (blue dashed) and SECS (External only) (grey dot-dash) method over a three-month period (April-June 2005) at magnetic observatory Iqaluit (IQA).

Numerical estimates of the fit are given in figure legends (and also Table 1). For example, the power of the data (Eq. 7) in the Y component is $62.8 \mathrm{nT}$, while the RMS misfit of the SECS and the SECS ( $\mathrm{X}$ and Y only) estimate is $40.2 \mathrm{nT}$ and the SECS (External only) estimate is $44.4 \mathrm{nT}$. The misfit of the latitudinal weighted estimate is $67.6 \mathrm{nT}$, indicating that the variance of the latitudinal weighted estimate is worse than that of the data. If one had assumed no change occurred in the field due to the external field throughout the day for this component, it would have been (slightly) better than using the latitudinal weighted estimate. Thus, in the Y component the SECS method shows a strong improvement on the latitudinal weighting method, though the advantage gained from the SECS method is not as great in the $\mathrm{X}$ and $\mathrm{Z}$ components.

In Network 4 (North Sea region), external field variations recorded at Eskdalemuir (ESK) observatory during the magnetically active day exceeded $150 \mathrm{nT}$. Network 4 contains four observatories and four variometers, two of which (Crooktree and York) are relatively close to Eskdalemuir. The latitudinal weighted estimate uses data from Lerwick (LER) and Hartland (HAR) observatories.

Figure 3 compares the estimated magnetic field from the SECS and latitudinal weighted methods. The SECS estimate most closely matches the measured data, especially in the $\mathrm{X}$ and $\mathrm{Z}$ components of the magnetic field where the largest variations are observed. In this case, the SECS method slightly underestimates the largest spikes seen in the magnetic field disturbance (e.g. between minutes 75 and 400 in the $\mathrm{X}$ component), but is otherwise a good fit. The latitudinal weighted estimate overestimates the magnitude of the changes in the field, while the SECS (External only) estimate again compares well in $\mathrm{X}$ and $\mathrm{Y}$ but is extremely poor in $\mathrm{Z}$. Note, the SECS ( $\mathrm{X}$ and $\mathrm{Y}$ only) estimate is very similar to the SECS estimate and is not shown.

The misfits of the SECS estimate are consistently smaller than the power of the data and provide a significant improvement over the latitudinal weighted estimate for this day. The SECS (External only) estimate indicates that modelling of the ionospheric current sheets alone cannot account for the observed magnetic field changes in the $\mathrm{Z}$ component.

The results of the analysis of power and misfit for all the networks are given in Table 1, which shows the RMS errors of the SECS, latitudinal-weighting, SECS (External only) and SECS (X and Y only) estimates for both the magnetically disturbed and magnetically quiet days. The generally smaller misfits of the SECS method in Table 1 indicate that improvements are possible when attempting to interpolate the field on magnetically disturbed days. However, on magnetically 


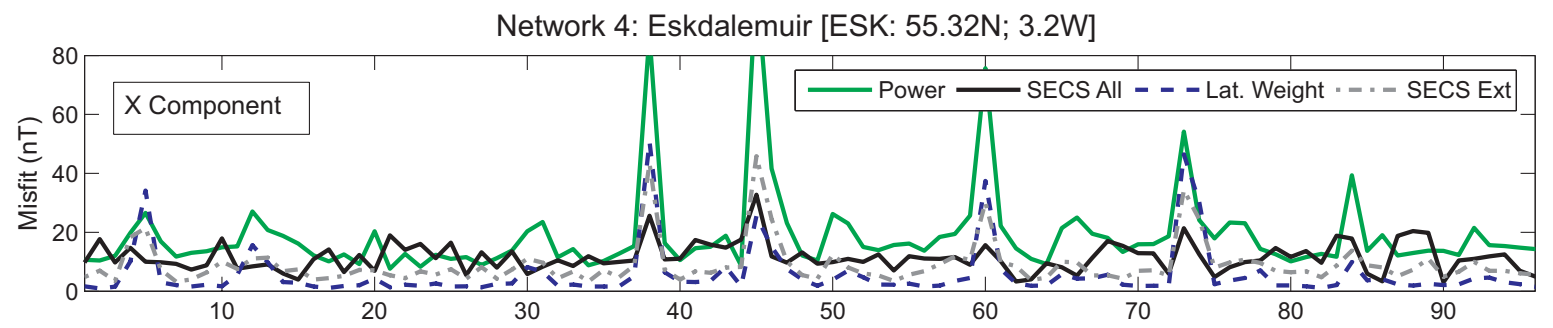

Y

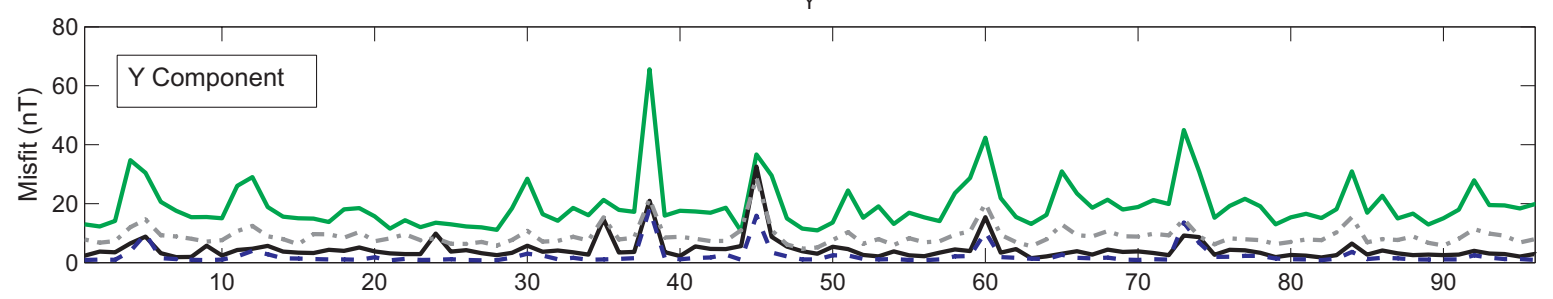

Z

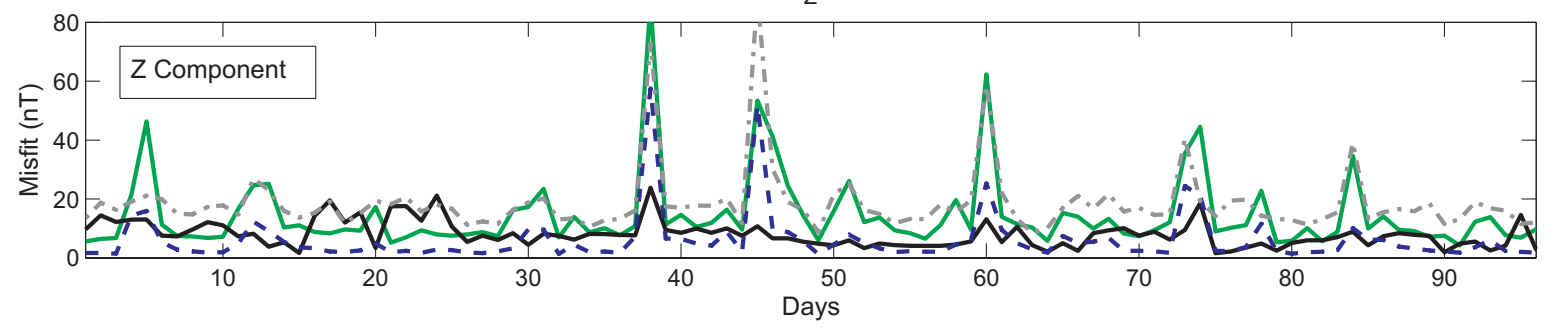

Fig. 5. Comparison of the daily power of measured external disturbances (green solid) with the misfit estimate from the SECS (black solid), Latitudinal Weighted (blue dashed) and SECS (External only) (grey dot-dash) method over a three-month period (April-June 2005) at magnetic observatory Eskdalemuir (ESK).

quiet days, the results of the latitudinal weighted method are comparable to the SECS method.

Scrutinising the results from Table 1 suggests that including a greater number of observatories (e.g. Network 1) lowers the misfit, even if these are uncalibrated variometers (as in Network 4). At lower geomagnetic latitudes (Network 5) improvements are not as marked. In Networks 2 and 3, which cover very large areas and have only five and seven observatories, respectively, the SECS estimates are not as good, particularly in the $\mathrm{Z}$ component on disturbed days. For Networks 1-4, the SECS (External only) estimate is extremely poor on disturbed days, again, particularly in the $\mathrm{Z}$ component suggesting that reasonable estimates of ground magnetic field disturbances require modelling of the induced magnetic field. By comparing the SECS (External only) mifits with the SECS ( $\mathrm{X}$ and $\mathrm{Y}$ only) misfits, it is clear that solving for the $\mathrm{X}$ and $\mathrm{Y}$ components using the external field part of the SECS equation (i.e. ignoring the $\mathrm{Z}$ component) greatly improves the estimate. However, in general, the SECS (X and $\mathrm{Y}$ only solution) misfits are not consistently smaller than the misfits from solving for the internal and external parts of the field together.

\subsection{Evaluation over three months}

Approximately three months of observatory and variometer data from the beginning of April to start of July 2005 were analysed, comprising of several quiet and relatively disturbed periods. A comparison of the daily power of the data and the RMS misfit of the SECS estimate, the latitudinal weighted estimate and the SECS (External only) estimate for Network 1 are shown in Fig. 4. The SECS method provides a consistent improvement to the estimate compared to the latitudinal weighting estimate. Results for Networks 2 and 3 are similar. This longer time series analysis illustrates that the SECS method is most beneficial on disturbed days, but on quieter days the latitudinal weighting is actually a good proxy for the magnetic field, even at widely separated longitudes. The SECS (External only) estimate has, in general, a slightly larger misfit to the observed data in all components.

The same period of 96 days of data was analysed for Network 4 . Figure 5 shows that both the SECS and the latitudinal weighting methods perform significantly better than predicting no external field change. The method of latitudinal weighting appears to perform better on days of small magnetic disturbance, while SECS is better during larger variations. On some days (e.g. Day 21-25) the SECS estimate 
Table 1. RMS errors of the Power, SECS, Latitudinal Weighting, SECS (External only) and SECS (X and Y only) estimates for the five networks, calculated over a period of twenty-four hours. Analysis is presented for a magnetically disturbed day (11 December 2005 ; 11 September 2005) and magnetically quiet day (7 December 2005; 21 September 2005), depending on the availability of data within each network. For Network 1, the observatories used for latitude weighting are GDH and NAQ; Network 2 uses CBB and FCC; Network 3 uses CBB and MEA; Network 4 uses LER and HAD; Network 5 uses WNG and AQU.

\begin{tabular}{|c|c|c|c|c|c|c|c|c|}
\hline Network & Obs. & Date & Comp. & Power & SECS & Lat. Wgt. & SECS (Ext only) & SECS (X;Y only) \\
\hline \multirow[t]{3}{*}{1} & IQA & $11 \mathrm{Dec} 2005$ & $X$ & 69.4 & 51.6 & 55.3 & 54.2 & 51.4 \\
\hline & & & $\mathrm{Y}$ & 62.8 & 40.2 & 67.6 & 44.4 & 40.2 \\
\hline & & & $\mathrm{Z}$ & 82.2 & 61.2 & 66.9 & 125.1 & - \\
\hline \multirow[t]{3}{*}{2} & BLC & $11 \mathrm{Dec} 2005$ & $\mathrm{X}$ & 69.7 & 59.7 & 78 & 52 & 60.7 \\
\hline & & & $\mathrm{Y}$ & 43.3 & 28.2 & 26.7 & 35.5 & 28.7 \\
\hline & & & $\mathrm{Z}$ & 81.9 & 65.1 & 68.4 & 123 & - \\
\hline \multirow[t]{3}{*}{3} & YKC & $11 \mathrm{Dec} 2005$ & $X$ & 114.9 & 52.5 & 94.6 & 55.1 & 54.7 \\
\hline & & & $\mathrm{Y}$ & 52.6 & 65.1 & 57.7 & 51.3 & 63.5 \\
\hline & & & $\mathrm{Z}$ & 103.7 & 97.2 & 115 & 133 & - \\
\hline \multirow[t]{3}{*}{4} & ESK & 11 Sep 2005 & $X$ & 56.2 & 13.7 & 34.8 & 25.9 & 13.4 \\
\hline & & & $\mathrm{Y}$ & 34.4 & 6.7 & 10.1 & 26.3 & 6.7 \\
\hline & & & $\mathrm{Z}$ & 68.2 & 7.7 & 24.8 & 73.6 & - \\
\hline \multirow[t]{3}{*}{5} & FUR & 11 Sep 2005 & $X$ & 49 & 8.6 & 7.1 & 24.1 & 8.2 \\
\hline & & & $\mathrm{Y}$ & 24.1 & 4.8 & 5.2 & 11.1 & 4.8 \\
\hline & & & $\mathrm{Z}$ & 25.3 & 5.2 & 8.8 & 11.3 & - \\
\hline \multirow[t]{3}{*}{1} & IQA & 7 Dec 2005 & $X$ & 12.6 & 7.5 & 10.5 & 8.2 & 7.6 \\
\hline & & & $\mathrm{Y}$ & 10.3 & 7.8 & 5.8 & 7.7 & 7.7 \\
\hline & & & $\mathrm{Z}$ & 12.8 & 10.5 & 11.6 & 12.3 & - \\
\hline \multirow[t]{3}{*}{2} & BLC & 7 Dec 2005 & $X$ & 21.6 & 16.9 & 23 & 15.7 & 17.3 \\
\hline & & & $\mathrm{Y}$ & 15.3 & 9.2 & 10 & 10.4 & 7.7 \\
\hline & & & $\mathrm{Z}$ & 17.8 & 18.4 & 19.4 & 18.2 & - \\
\hline \multirow[t]{3}{*}{3} & YKC & 7 Dec 2005 & $X$ & 8.9 & 6.3 & 12.1 & 6.8 & 6.5 \\
\hline & & & $\mathrm{Y}$ & 6.7 & 4.7 & 9.2 & 5 & 4.6 \\
\hline & & & Z & 7.7 & 8.3 & 13.7 & 21.7 & - \\
\hline \multirow[t]{3}{*}{4} & ESK & 21 Sep 2005 & $\mathrm{X}$ & 9.5 & 12.3 & 1.3 & 3.3 & 12.4 \\
\hline & & & $\mathrm{Y}$ & 9.3 & 1.6 & 0.6 & 4.3 & 1.6 \\
\hline & & & $\mathrm{Z}$ & 4.5 & 7.6 & 2.1 & 22.3 & - \\
\hline \multirow[t]{3}{*}{5} & FUR & 21 Sep 2005 & $X$ & 7.4 & 2 & 1 & 4.2 & 1.9 \\
\hline & & & $\mathrm{Y}$ & 11.8 & 1.3 & 0.5 & 5.5 & 1.2 \\
\hline & & & $\mathrm{Z}$ & 4.3 & 0.7 & 1.5 & 3.6 & - \\
\hline
\end{tabular}

is worse than assuming no external field change, which may be due to the inclusion of uncalibrated variometers, as the estimate misfit rarely falls below about $15 \mathrm{nT}$ in the $\mathrm{X}$ and $\mathrm{Z}$ components. Interestingly, the SECS (External only) estimate has a lower average misfit in the $\mathrm{X}$ component, though is markedly poorer in $\mathrm{Z}$.

\section{Discussion}

The external magnetic field and its influence on the ground magnetic field are still relatively poorly understood. The SECS method provides a flexible approach to modelling ionospheric equivalent currents and is potentially useful in space weather applications, for example. Previous experiments using the SECS method have been primarily conducted with dense magnetometer arrays, such as the BEAR and IMAGE networks in Scandinavia (e.g. Pulkkinen et al., 2003a; Viljanen et al., 2004). It is certainly reasonable to assume that these array configurations are better suited to SECS. However, we have shown that the SECS method is similarly applicable in locations where magnetic measurements are sparsely distributed over large regions. This is specifically where other more developed methods such as the Fourier method or SCHA have been shown to be insufficient (Amm and Viljanen, 1999). Recently, the SECS technique 
has also been applied in the analysis of magnetospheric and ionospheric flow vortices with a large set of magnetometer stations in northern America, from Alaska to Greenland, involving a number of magnetometer arrays including THEMIS and CARISMA in combination with satellite-based observations (Keiling et al., 2009).

The improved estimate of the field from the SECS interpolation method is very noticeable at high geomagnetic latitudes. The SECS estimate of the field is generally better than latitudinal weighting particularly during magnetically disturbed conditions. In magnetically quiet conditions the latitudinal weighting estimate is comparable or occasionally better. At lower geomagnetic latitudes, the SECS method again produces the lowest misfit during magnetically active conditions but performs less well in very quiet conditions. Modelling the magnetic field using only the external elementary current systems produced misfits which were consistently poorer. This indicates that solving for internal current systems greatly improves the resulting estimate from the SECS method, particularly in the $\mathrm{Z}$ component.

Clearly, compared to latitudinal-based interpolation based on data from two observatories, there is a greater logistical and computational cost in acquiring data from a larger number of observatories to use with the SECS method e.g. for real-time or practical purposes. However, if the data are available, the benefits from the improved estimate, particularly during noisy conditions, outweigh the slightly larger computational costs, as the inversion of the magnetic field data using Singular Value Decomposition and computation of the $\mathbf{T}$ matrix are extremely fast.

As a more sophisticated external field interpolation approach, we suggest it could have applications in marine and aeromagnetic surveying, improving the ability to resolve the local crustal field, or perhaps in satellite magnetometry to remove the effects of ionospheric currents for more accurate main field models. Other applications include better realtime estimates of the field for directional drilling and potential prediction of geomagnetically-induced currents in power systems.

\section{Conclusions}

Interpolation of the magnetic field over large areas can be achieved using Spherical Elementary Current Systems. The method is derived from Maxwell's equations and represents a physical rather than a mathematical approach to interpolation and extrapolation. We show that the method can be applied to produce good estimates of the magnetic field over large regions using data from a relatively small number of observatories. In addition to modelling external field variations, we found that including ground induced current systems gave a marked improvement in the estimate of the $\mathrm{Z}$ component of the magnetic field.
At high geomagnetic latitudes, the SECS method gave the best estimate of the external magnetic field, particularly during magnetically active days. At lower latitudes, during magnetically quiet periods, the SECS method is comparable to the latitudinal weighted average of two observatories, one approximately to the north and the other to the south of the point of interest.

We have shown that the SECS method can be applied to networks of different sizes where various densities of observatory data are available. In situations where direct measurement of the external field is not possible due to logistical or physical constraints we suggest the SECS method can be used as a powerful interpolation tool.

Acknowledgements. We wish to acknowledge Allan McKay for development and initial coding of the algorithms used in this study. We thank O. Amm for his thorough review and suggestions for improving the manuscript. The institutes that support magnetic observatories together with INTERMAGNET are thanked for promoting high standards of observatory practice. INTERMAGNET data was obtained from the World Data Centre (Edinburgh). The SubAuroral Magnetometer Network data (SAMNET) is operated by the Department of Communications Systems at Lancaster University (UK) and is funded by the Science and Technology Facilities Council (STFC). This paper is published with the permission of the Executive Director of the British Geological Survey (NERC).

Topical Editor K. Kauristie thanks O. Amm for his help in evaluating this paper.

\section{References}

Amm, O.: Ionospheric elementary current systems in spherical coordinates and their application, J. Geomag. Geoelectr., 49, 947955, 1997.

Amm, O. and Viljanen, A.: Ionospheric disturbance magnetic field continuation from the ground to the ionosphere using spherical elementary current systems, Earth Planets Space, 51, 431-440, 1999.

Boteler, D., Pirjola, R., and Nevanlinna, H.: The effects of geomagnetic disturbances on electrical systems at the Earth's surface, Adv. Space Res., 22, 17-27, 1998.

Bowe, J. and McCulloch, S.: Space Weather, chap. The value of real-time geomagnetic reference data to the oil and gas industry, pp. 289-298, Springer, 2007.

Campbell, W.: Introduction to Geomagnetic Fields, Cambridge University Press., 2003.

Haines, G.: Spherical cap harmonic analysis, J. Geophys. Res., 90, 2583-2591, 1985.

Keiling, A., Angelopoulos, V. Runov, A., Weygand, J., Apatenkov, S. V., Mende, S., McFadden, J., Larson, D., Amm, O., Glassmeier, K.-H., and Auster, H. U.: Substorm current wedge driven by plasma flow vortices: THEMIS observations, J. Geophys. Res., 114, A00C22, doi:10.1029/2009JA014114, 2009.

Kerridge, D.: INTERMAGNET: Worldwide near-real-time geomagnetic observatory data, Proceedings of the Workshop on Space Weather, ESTEC, 2001. 
Klobuchar, J.: Ionospheric time-delay algorithm for singlefrequency GPS users, IEEE Transactions on Aerospace and Electronics System, 23, 325-331, 1986.

Mersmann, U., Baumjohann, W., Küppers, F., and Lange, K.: Analysis of an Eastward Electrojet by means of upward continuation of ground-based magnetometer data, J. Geophys., 45, 281-298, 1979.

Pirjola, R.: Effects of space weather on high-latitude ground systems, Adv. Space Res., 36, 2231-2240, 2005.

Pirjola, R. and Viljanen, A.: Complex image method for calculating electric and magnetic fields produced by an auroral electrojet of finite length, Ann. Geophys., 16, 1434-1444, doi:10.1007/s00585-998-1434-6, 1998.

Pirjola, R., Viljanen, A., Pulkkinen, A., and Amm, O.: Space weather risk in power systems and pipelines, Phys. Chem. Earth, 25, $333-337,2000$.

Pulkkinen, A., Amm, O., Viljanen, A., and BEAR Working Group: Separation of the geomagnetic field variation on the ground into external and internal parts using the spherical elementary current system method, Earth Planets Space, 55, 117-129, 2003a.

Pulkkinen, A., Amm, O., Viljanen, A., and BEAR Working Group: Ionospheric equivalent current distributions determined with the method of spherical elementary current systems, J. Geophys. Res., 108, 1053, doi:10.1029/2001JA005085, 2003 b.
Reay, S. J., Allen, W., Baillie, O., Bowe, J., Clarke, E., Lesur, V., and Macmillan, S.: Space weather effects on drilling accuracy in the North Sea, Ann. Geophys., 23, 3081-3088, doi:10.5194/angeo-23-3081-2005, 2005.

Ritter, P., Lühr, H., Viljanen, A., Amm, O., Pulkkinen, A., and Sillanpää, I.: Ionospheric currents estimated simultaneously from CHAMP satelliteand IMAGE ground-based magnetic field measurements: a statisticalstudy at auroral latitudes, Ann. Geophys., 22, 417-430, doi:10.5194/angeo-22-417-2004, 2004.

Tanskanen, E. I., Viljanen, A., Pulkkinen, L., Pirjola, R., Häkkinen, L., Pulkkinen, A., and Amm, O.: At substorm onset, 40\% of AL comes from underground, J. Geophys. Res., 106, 13119-13134, 2001.

Vanhamäki, H., Amm, O., and Viljanen, A.: One-dimensional upward continuation of the ground magnetic field disturbance using spherical elementary current systems, Earth Planets Space, 55, 613-625, 2003.

Viljanen, A., Pulkkinen, A., Amm, O., Pirjola, R., Korja, T., and BEAR Working Group: Fast computation of the geoelectric field using the method of elementary current systems and planar Earth models, Ann. Geophys., 22, 101-113, doi:10.5194/angeo-22101-2004, 2004. 